\title{
A quantum cloning bound and application to quantum key distribution
}

\author{
Erik Woodhead* \\ Laboratoire d'Information Quantique, CP 225, Université Libre de \\ Bruxelles (ULB), Boulevard du Triomphe, 1050 Brussels, Belgium
}

14 August 2013

\begin{abstract}
We introduce a quantum cloning bound which we apply to a straightforward and relatively direct security proof of the prepare-and-measure Bennett-Brassard 1984 (BB84) quantum key distribution (QKD) protocol against collective attacks. The approach we propose is able to handle the practical problem of source and detector alignment imprecisions in a simple way. Specifically, we derive a keyrate bound for a BB84 implementation in which Alice's source emits four given but arbitrary pure states, where the usual equivalence between prepareand-measure and entanglement-based QKD no longer applies. Our result is similar to a keyrate derived by Marøy et al. [Phys. Rev. A 82, 032337 (2010)] and generally an improvement over the keyrate derivable from the entropic uncertainty relation in situations where it applies. We also provide a stronger result for a source emitting arbitrary qubit states, and a further improved result if the detector is additionally assumed two dimensional.
\end{abstract}

\footnotetext{
${ }^{*}$ Erik.Woodhead@ulb.ac.be
} 


\section{Introduction}

\subsection{Background and motivation}

Quantum key distribution (QKD) [1] is an approach to the problem of generating a cryptographic key and disseminating it securely between its intended recipients. The general advantage of QKD is that it permits the security of a key to be certified based only on the laws of physics, in particular limitations inherent to quantum physics, rather than assumptions about the computational power of a potential adversary.

There are two related classes of QKD implementation considered in the literature: the entanglement-based approach [2], in which the secret key is generated by joint measurements on a shared entangled state, and "prepareand-measure" QKD [1], in which one party ("Alice") prepares states and transmits them to another ("Bob"). A simple well-known argument [3] establishes a correspondence between entanglement-based protocols and a restricted class of prepare-and-measure protocols in which the source states satisfy a condition of basis independence [4] (where the average of the source states is the same in the different bases used).

While this argument has played an important role in the development of security proofs, it also has its limitations. Real world QKD implementations are subject to various practical issues, including imperfections in the devices, which can lead to a false certification of security if not accounted for in the security analysis [5]. In particular, most experimental and commercially available QKD implementations are of the prepare-and-measure variety. In this case the states used in the implementation will not form orthogonal bases and will inevitably deviate from the basis-independence condition due to the limited accuracy of the source. Consequently, security proofs of entanglement-based QKD protocols accounting for arbitrary detector alignment imprecisions - such as the recent security proofs of the entanglement-based Bennett-Brassard (BB84) BB84 protocol based on entropic uncertainty relations [6-8] - do not translate to security proofs accounting for all possible source alignment flaws in the prepare-and-measure setting.

A few security proofs of the prepare-and-measure BB84 protocol have tackled this issue based on a reduction to an equivalent virtual protocol [9-11]. In this virtual protocol, Alice's bit and basis choices are effectively both determined by the outcome of a single four-outcome measurement on an entangled "coin" state that must be partially or fully characterised and pre- 
pared by Alice. Conceptually, this differs from security analyses of genuine entanglement-based QKD protocols, which assume the entangled state is prepared by an adversary and unknown to the legitimate parties apart from what they can deduce about it via their measurement results.

In this paper, we propose an alternative approach to this problem in which we abandon the use of entangled states altogether. We show that a straightforward and relatively direct asymptotic security proof of the prepare-andmeasure BB84 protocol against collective attacks is possible based on an appropriate characterisation of the limitations of quantum cloning. The nocloning theorem [12, 13], which implies that an eavesdropper cannot copy non-orthogonal states without disturbing them in a visible way, is often considered the intuitive basis for the security of QKD. This intuitive connection was noted in the original proposal of Bennett and Brassard [1], and is further discussed in [14]. The approach of studying the prepare-and-measure BB84 protocol "directly" was followed in some early generic security analyses $[15,16]$, including the original security proof of BB84 against individual attacks [17], though has seen little development following Shor and Preskill's security proof based on entanglement distillation [18].

As our main result, we derive a secret keyrate for a BB84 implementation in which the source emits four given but arbitrary pure states and which is largely device independent on Bob's side. Our end result is comparable to the keyrate derived by Marøy et al. [11] which, to our knowledge, is the only previous analysis that establishes security under such relaxed conditions. Specifically, we recover the same keyrate as Marøy et al. if the two $z$-basis source states are emitted with equal probability, but show that it can be improved in the case of a biased source.

While it is interesting to have a security result that holds for arbitrary source states, there are situations of practical interest where these states are naturally qubits. This is the case if, for instance, Alice encodes her basis and bit choices in the form of different photon polarisation states. In such an implementation, the source states may still span a Hilbert space of more than two dimensions in practice if, for example, an attenuated laser is used to produce low intensity coherent states. Anticipating that this complication is probably best addressed with the decoy-state method [19, 20], as a second main result we also present an improved keyrate bound for a source emitting arbitrary qubit states. If we additionally assume Bob's detector measurements are two dimensional, we find that we can further improve our result to the point that, if no errors are observed, we obtain an asymptotic keyrate of 1 . 


\subsection{Outline of scenario and main results}

We imagine Alice possesses a source which, depending on a choice of bit and "basis", emits one of four different quantum states which is sent to Bob. Throughout this paper we will consistently call the two " $z$-basis" states $|\alpha\rangle$ and $\left|\alpha^{\prime}\right\rangle$, and the two " $x$-basis" states $|\beta\rangle$ and $\left|\beta^{\prime}\right\rangle$ (note that this nomenclature is not intended to imply that these states are necessarily really the $\sigma_{z}$ and $\sigma_{x}$ eigenstates). We denote the corresponding density operators by $\rho=|\alpha\rangle\left\langle\alpha\left|, \rho^{\prime}=\right| \alpha^{\prime}\right\rangle\left\langle\alpha^{\prime}|, \sigma=| \beta\right\rangle\langle\beta|$, and $\sigma^{\prime}=\left|\beta^{\prime}\right\rangle\left\langle\beta^{\prime}\right|$. Following a unitary attack by a potential eavesdropper ("Eve"), these states are contained in some Hilbert space $\mathcal{H}_{\mathrm{A}} \subset \mathcal{H}_{\mathrm{B}} \otimes \mathcal{H}_{\mathrm{E}}$, where $\mathcal{H}_{\mathrm{B}}$ and $\mathcal{H}_{\mathrm{E}}$ respectively denote the Hilbert spaces accessible to Bob and Eve. Upon reception of the states emitted by Alice, Bob performs one of two binary-outcome measurements (a "z-basis" or " $x$-basis" measurement, though we do not assume Bob is necessarily measuring $\sigma_{z}$ or $\sigma_{x}$ ) in order to compute his version of the raw key. Alice and Bob then sacrifice a subset of their results in order to estimate the $z$ - and $x$-basis bit error rates $\delta_{z}$ and $\delta_{x}$, and if these are not too high, extract a secret key by error correction and privacy amplification.

We consider the common variant of $\mathrm{BB} 84$ where only the $z$-basis results are used to generate the key. In this case, for an unbiased source an application of the Devetak-Winter bound [21] yields a lower bound on the asymptotic secret keyrate $r$ which depends only on the fidelity $F\left(\rho_{\mathrm{E}}, \rho_{\mathrm{E}}^{\prime}\right)$ between the parts of the $z$-basis states accessible to the eavesdropper:

$$
r \geq 1-h\left(\frac{1}{2}+\frac{1}{2} F\left(\rho_{\mathrm{E}}, \rho_{\mathrm{E}}^{\prime}\right)\right)-h\left(\delta_{z}\right) .
$$

In this expression, the fidelity is defined by $F(\rho, \sigma)=\|\sqrt{\rho} \sqrt{\sigma}\|_{1}$ where $\|\cdot\|_{1}$ denotes the trace norm, $h(x)=-x \log (x)-(1-x) \log (1-x)$ is the binary entropy, all logarithms are in base 2 , and subscripts indicate partial tracing in the usual way (e.g. $\rho_{\mathrm{E}}=\operatorname{Tr}_{\mathrm{B}}[\rho]$ ). Bounding the keyrate is then reduced to lower bounding $F\left(\rho_{\mathrm{E}}, \rho_{\mathrm{E}}^{\prime}\right)$. Alice and Bob, by contrast, may lower bound the trace distances $D\left(\rho_{\mathrm{B}}, \rho_{\mathrm{B}}^{\prime}\right)$ and $D\left(\sigma_{\mathrm{B}}, \sigma_{\mathrm{B}}^{\prime}\right)$ in terms of the observed bit error rates via the Helstrom bound [22]: $D\left(\rho_{\mathrm{B}}, \rho_{\mathrm{B}}^{\prime}\right) \geq\left|1-2 \delta_{z}\right|$ and $D\left(\sigma_{\mathrm{B}}, \sigma_{\mathrm{B}}^{\prime}\right) \geq$ $\left|1-2 \delta_{x}\right|$, where we define the trace distance by $D(\rho, \sigma)=\frac{1}{2}\|\rho-\sigma\|_{1}$. Our derivation is then completed by bounding $F\left(\rho_{\mathrm{E}}, \rho_{\mathrm{E}}^{\prime}\right)$ in terms of $D\left(\sigma_{\mathrm{B}}, \sigma_{\mathrm{B}}^{\prime}\right)$. Any such bound can be understood as a fundamental limit on the degree to which quantum states can be cloned.

In general, such a bound will depend on a characterisation of the states $|\alpha\rangle$, $\left|\alpha^{\prime}\right\rangle,|\beta\rangle$, and $\left|\beta^{\prime}\right\rangle$ emitted by the source. Similar to the authors of $[9,11]$, we will consider the case where we are given a "basis overlap" angle $\theta$, which we define such that

$$
\sqrt{1+|\sin (\theta)|}=\frac{1}{2}\left|\langle\alpha \mid \beta\rangle+\left\langle\alpha^{\prime} \mid \beta\right\rangle+\left\langle\alpha \mid \beta^{\prime}\right\rangle-\left\langle\alpha^{\prime} \mid \beta^{\prime}\right\rangle\right|
$$


wherever the right-hand side is greater than 1. Our main cloning bound then reads

$$
F\left(\rho_{\mathrm{E}}, \rho_{\mathrm{E}}^{\prime}\right) \geq f_{\theta}\left(D\left(\sigma_{\mathrm{B}}, \sigma_{\mathrm{B}}^{\prime}\right)\right),
$$

with the function $f_{\theta}$ defined by

$$
f_{\theta}(v)=\left\{\begin{array}{ll}
|\sin (\theta)| v-|\cos (\theta)| \sqrt{1-v^{2}} & : v \geq|\cos (\theta)| \\
0 & : v \leq|\cos (\theta)|
\end{array},\right.
$$

which provides a lower bound on $F\left(\rho_{\mathrm{E}}, \rho_{\mathrm{E}}^{\prime}\right)$ wherever the right-hand side of (2) is greater than 1.

These are the essential ingredients of our security analysis. Bounding the keyrate is a matter of applying (3) and $D\left(\sigma_{\mathrm{B}}, \sigma_{\mathrm{B}}^{\prime}\right) \geq\left|1-2 \delta_{x}\right|$ to (1). The resulting keyrate is then

$$
r \geq 1-h\left(\frac{1}{2}+\frac{1}{2} f_{\theta}\left(\left|1-2 \delta_{x}\right|\right)\right)-h\left(\delta_{z}\right) .
$$

This coincides with the keyrate given in [11] for the scenario we have outlined here, though as will be discussed later on, our general approach can lead to improved keyrates in certain cases, specifically where the source is biased or is limited to two dimensions and more information about the states is used.

We give a derivation of the asymptotic keyrate bound (1) for an unbiased source in section 2, as well as a generalisation for a biased source where the two $z$-basis states are not emitted with equal probability. We also briefly comment on the issue of non-asymptotic security, which we mostly leave as a problem for future work. In section 3, we discuss how an eavesdropper's distinguishing power between the two $z$-basis states, measured in terms of the fidelity as described above, can be bounded. This includes a proof of the cloning bound (3) introduced above, a better bound applicable if the source states are qubits, and a further improved bound if both the source and detector are two dimensional. We finish with some concluding remarks and summarise potential directions for future research in section 4 .

\section{Bounding the keyrate}

\subsection{Derivation of asymptotic keyrate bound}

Assuming an eavesdropper restricted to collective attacks, a lower bound on the asymptotic secret keyrate that can be securely extracted from Alice's 
sifted key by one-way error correction and privacy amplification is given by the Devetak-Winter bound [21]:

$$
r \geq H(Z \mid \mathrm{E})-H\left(Z \mid Z^{\prime}\right) .
$$

Here, the conditional Shannon entropy $H\left(Z \mid Z^{\prime}\right)$ quantifies the key loss due to error correction, and is upper bounded by $h\left(\delta_{z}\right)$ (with equality in the typical case of symmetric errors). The conditional von Neumann entropy $H(Z \mid$ E) quantifies the amount of key that can be extracted securely by privacy amplification, which for our purposes we should evaluate on the classical-quantum state

$$
\tau_{Z \mathrm{E}}=\frac{1}{2}\left(|0\rangle\left\langle\left. 0\right|_{Z} \otimes \rho_{\mathrm{E}}+\mid 1\right\rangle\left\langle\left. 1\right|_{Z} \otimes \rho_{\mathrm{E}}^{\prime}\right),\right.
$$

where $\rho$ and $\rho^{\prime}$ are the $z$-basis states emitted by Alice, and $|0\rangle_{Z}$ and $|1\rangle_{Z}$ denote the state of a classical register in her possession. The state (7) expresses the correlation between Alice's version of the sifted key and the corresponding quantum states in Eve's possession.

In order to arrive at (1), we use that $H(Z \mid \mathrm{E}) \geq H\left(Z \mid \mathrm{EE}^{\prime}\right)$ for any extension of the state $(7)$ to a larger Hilbert space $\mathcal{H}_{Z} \otimes \mathcal{H}_{\mathrm{E}} \otimes \mathcal{H}_{\mathrm{E}^{\prime}}$ (formally this follows from the property of strong subadditivity of the von Neumann entropy). In particular, we use this to replace $\rho_{\mathrm{E}}$ and $\rho_{\mathrm{E}}^{\prime}$ in (7) with purifications $|\Psi\rangle$ and $|\Phi\rangle \in \mathcal{H}_{\mathrm{E}} \otimes \mathcal{H}_{\mathrm{E}^{\prime}}$. Applying the definition of the conditional von Neumann entropy, we find

$$
\begin{aligned}
H(Z \mid \mathrm{E}) & \geq H\left(Z \mid \mathrm{EE}^{\prime}\right) \\
& =1-S\left[\frac{1}{2}(|\Psi\rangle\langle\Psi|+| \Phi\rangle\langle\Phi|)\right] \\
& =1-h\left[\frac{1}{2}(1+|\langle\Psi \mid \Phi\rangle|)\right],
\end{aligned}
$$

where $S(\rho)=-\operatorname{Tr}[\rho \log (\rho)]$. Since by Uhlmann's theorem we can choose $|\Psi\rangle$ and $|\Phi\rangle$ such that $F\left(\rho_{\mathrm{E}}, \rho_{\mathrm{E}}^{\prime}\right)=|\langle\Psi \mid \Phi\rangle|$, we find

$$
H(Z \mid \mathrm{E}) \geq 1-h\left(\frac{1}{2}+\frac{1}{2} F\left(\rho_{\mathrm{E}}, \rho_{\mathrm{E}}^{\prime}\right)\right),
$$

which completes the derivation of (1). Note that (9) is equivalent to an analogous bound on the Holevo quantity derived in [23].

In the proof given here, we have made the tacit assumption that Alice emits the $z$ states $|\alpha\rangle$ and $\left|\alpha^{\prime}\right\rangle$ with equal probability. If we wish to account for the possibility of a biased source, as was considered in [11] for instance, then it is straightforward to adapt the derivation of (1). In particular, if the two $z$-basis states are emitted with probabilities $p=(1+\varepsilon) / 2$ and $q=(1-\varepsilon) / 2$ respectively, (9) generalises to

$$
H(Z \mid \mathrm{E}) \geq h(p)-h\left[\frac{1}{2}+\frac{1}{2} \sqrt{\varepsilon^{2}+\left(1-\varepsilon^{2}\right) F\left(\rho_{\mathrm{E}}, \rho_{\mathrm{E}}^{\prime}\right)^{2}}\right] .
$$


Our results will hold essentially unmodified if there is a bias in the $x$ basis, provided the observed coincidence statistics are used to correctly bound the trace distance $D\left(\sigma_{\mathrm{B}}, \sigma_{\mathrm{B}}^{\prime}\right)$. Here we have departed from the approach used by the authors of [9-11], where any bias in the source was included in their analogue of our source characterisation (2). This way we obtain a generally better result. For instance, for an otherwise ideal BB84 implementation and in the absence of errors, we obtain the best possible keyrate of $r=h(p)$.

\subsection{A note on non-asymptotic security}

As alluded to above, the Devetak-Winter bound, and consequently the bound (1) on the keyrate that we will use in the remainder of this paper, is only guaranteed to hold in the asymptotic limit of an infinitely long key. In the realistic finite-key case, a finite-key result can be derived based on a "one shot" version of the Devetak-Winter rate [7] where the main problem in obtaining a security proof is reduced to lower bounding the conditional min-entropy $H_{\min }(Z \mid$ E) rather than the von Neumann entropy $H(Z \mid \mathrm{E})$. To obtain a result that converges asymptotically to the keyrate given by the Devetak-Winter bound, the min-entropy should ideally be evaluated with a smoothing parameter, which can roughly be interpreted as a small additional probability of failure traded in exchange for a higher keyrate, and which can be made arbitrarily small in the asymptotic limit. We refer to $[8,24]$ for a discussion of the details.

We will leave as a problem for future work how the smooth min-entropy can be bounded in such a way as to recover (9) asymptotically. We note only here that the min-entropy (without smoothing) evaluated on the state (7) has an exact expression in terms of the trace distance:

$$
H_{\min }(Z \mid \mathrm{E})=1-\log \left(1+D\left(\rho_{\mathrm{E}}, \rho_{\mathrm{E}}^{\prime}\right)\right) .
$$

This is a special case of the min-entropy's expression in terms of the guessing probability [25]; we also gave a simple derivation in [26]. Using that $D\left(\rho_{\mathrm{E}}, \rho_{\mathrm{E}}^{\prime}\right) \leq \sqrt{1-F\left(\rho_{\mathrm{E}}, \rho_{\mathrm{E}}^{\prime}\right)^{2}}$, we can alternatively bound the min-entropy in terms of the fidelity. While the result would be less than optimal and still limited to collective attacks, it is worth noting that using (11) in place of (9) would already allow the obtention of finite-key bounds using the general approach explored in this paper. 


\section{Bounding Eve's distinguishing ability}

\subsection{Ideal BB84 source}

In the special case of an ideal BB84 source, characterised by $\theta=\pi / 2$, the general cloning bound (3) reduces to

$$
F\left(\rho_{\mathrm{E}}, \rho_{\mathrm{E}}^{\prime}\right) \geq D\left(\sigma_{\mathrm{B}}, \sigma_{\mathrm{B}}^{\prime}\right),
$$

and we recover the famous keyrate $r \geq 1-h\left(\delta_{x}\right)-h\left(\delta_{z}\right)$ due to Shor and Preskill [18]. While the proof of (3) is not complicated, a direct proof of (12) is especially simple and will serve to introduce our techniques.

In an ideal BB84 implementation, the source states form two mutually unbiased orthogonal bases, i.e. satisfy

$$
\left\langle\alpha \mid \alpha^{\prime}\right\rangle=\left\langle\beta \mid \beta^{\prime}\right\rangle=0
$$

and, in an appropriate phase convention,

$$
\begin{aligned}
|\alpha\rangle & =\frac{1}{\sqrt{2}}\left[|\beta\rangle+\left|\beta^{\prime}\right\rangle\right], \\
\left|\alpha^{\prime}\right\rangle & =\frac{1}{\sqrt{2}}\left[|\beta\rangle-\left|\beta^{\prime}\right\rangle\right] .
\end{aligned}
$$

Equivalently, one can identify $Z=\rho-\rho^{\prime}$ and $X=\sigma-\sigma^{\prime}$ with the Pauli $z$ and $x$ operators. With this notation, $D\left(\rho_{\mathrm{B}}, \rho_{\mathrm{B}}^{\prime}\right)=\frac{1}{2}\left\|Z_{\mathrm{B}}\right\|_{1}$ and $D\left(\sigma_{\mathrm{B}}, \sigma_{\mathrm{B}}^{\prime}\right)=$ $\frac{1}{2}\left\|X_{\mathrm{B}}\right\|_{1}$.

We obtain (12) as follows. Let $U_{\mathrm{B}}$ be the (Hermitian) unitary operator acting on $\mathcal{H}_{\mathrm{B}}$ such that $\frac{1}{2}\left\|X_{\mathrm{B}}\right\|_{1}=\frac{1}{2} \operatorname{Tr}_{\mathrm{B}}\left[U_{\mathrm{B}} X_{\mathrm{B}}\right]$. Then,

$$
\begin{aligned}
D\left(\sigma_{\mathrm{B}}, \sigma_{\mathrm{B}}^{\prime}\right) & =\frac{1}{2} \operatorname{Tr}_{\mathrm{B}}\left[U_{\mathrm{B}} X_{\mathrm{B}}\right] \\
& =\frac{1}{2} \operatorname{Tr}\left[\left(U_{\mathrm{B}} \otimes \mathbb{1}_{\mathrm{E}}\right) X\right] \\
& =\frac{1}{2}\left(\left\langle\alpha\left|U_{\mathrm{B}} \otimes \mathbb{1}_{\mathrm{E}}\right| \alpha^{\prime}\right\rangle+\left\langle\alpha^{\prime}\left|U_{\mathrm{B}} \otimes \mathbb{1}_{\mathrm{E}}\right| \alpha\right\rangle\right) \\
& =\operatorname{Re}\left[\left\langle\alpha\left|U_{\mathrm{B}} \otimes \mathbb{1}_{\mathrm{E}}\right| \alpha^{\prime}\right\rangle\right] \\
& \leq\left|\left\langle\alpha\left|U_{\mathrm{B}} \otimes \mathbb{1}_{\mathrm{E}}\right| \alpha^{\prime}\right\rangle\right| .
\end{aligned}
$$

Now, $|\alpha\rangle$ and $\left|\alpha^{\prime}\right\rangle$ are by definition purifications of $\rho_{\mathrm{E}}$ and $\rho_{\mathrm{E}}^{\prime}$, and since $U_{\mathrm{B}} \otimes$ $\mathbb{1}_{\mathrm{E}}$ acts nontrivially only on $\mathcal{H}_{\mathrm{B}}$, the state $U_{\mathrm{B}} \otimes \mathbb{1}_{\mathrm{E}}\left|\alpha^{\prime}\right\rangle$ is still a purification of $\rho_{\mathrm{E}}^{\prime}$. Thus, by Uhlmann's theorem, the last line of (15) provides a lower bound on the fidelity between $\rho_{\mathrm{E}}$ and $\rho_{\mathrm{E}}^{\prime}$, which concludes our proof.

We conclude this discussion of the ideal BB84 scenario by noting that (12) can be seen as a strengthened version of a bound originally derived in [17]. Using that $D\left(\rho_{\mathrm{E}}, \rho_{\mathrm{E}}^{\prime}\right) \leq \sqrt{1-F\left(\rho_{\mathrm{E}}, \rho_{\mathrm{E}}^{\prime}\right)^{2}},(12)$ implies

$$
D\left(\rho_{\mathrm{E}}, \rho_{\mathrm{E}}^{\prime}\right)^{2}+D\left(\sigma_{\mathrm{B}}, \sigma_{\mathrm{B}}^{\prime}\right)^{2} \leq 1 .
$$


Although not given in this form, Fuchs et al. effectively derived this relation as an intermediate result in their security proof of the BB84 protocol against individual attacks (specifically, (16) is essentially equivalent to equations (23) and (24) in [17]).

\subsection{Arbitrary source states}

We now derive (3). Our starting point is the quantity

$$
\Delta=\frac{1}{2 \sqrt{2}}\left(\langle\alpha \mid \beta\rangle+\left\langle\alpha^{\prime} \mid \beta\right\rangle+\left\langle\alpha \mid \beta^{\prime}\right\rangle-\left\langle\alpha^{\prime} \mid \beta^{\prime}\right\rangle\right) .
$$

Note that (17) as expressed above is dependent on physically irrelevant phase factors (e.g. $|\alpha\rangle$ and $\mathrm{e}^{\mathrm{i} \phi}|\alpha\rangle$ denote the same state); to obtain the best result, one should adopt the phase convention that maximises (17), which can then always be taken to be real.

Let $P$ and $Q$ be orthogonal projectors such that $P-Q=U$ and $P+Q=\mathbb{1}$, where $U=U_{\mathrm{B}} \otimes \mathbb{1}_{\mathrm{E}}$ is the Hermitian unitary such that $\frac{1}{2} \operatorname{Tr}\left[U\left(\sigma-\sigma^{\prime}\right)\right]=$ $D\left(\sigma_{B}, \sigma_{\mathrm{B}}^{\prime}\right)$, as in the proof of (12). For convenience, we also define the (generally not normalised) states $\left|\alpha_{ \pm}\right\rangle=\left(|\alpha\rangle \pm\left|\alpha^{\prime}\right\rangle\right) / \sqrt{2}$. Then,

$$
\begin{aligned}
2 \Delta= & \left\langle\alpha_{+}|(P+Q)| \beta\right\rangle+\left\langle\alpha_{-}|(P+Q)| \beta^{\prime}\right\rangle \\
\leq & \left|\left\langle\alpha_{+}|P| \beta\right\rangle\right|+\left|\left\langle\alpha_{+}|Q| \beta\right\rangle\right| \\
& +\left|\left\langle\alpha_{-}|P| \beta^{\prime}\right\rangle\right|+\left|\left\langle\alpha_{-}|Q| \beta^{\prime}\right\rangle\right| .
\end{aligned}
$$

Applying the Cauchy-Schwarz inequality twice on the first and fourth terms on the right-hand side,

$$
\begin{aligned}
& \left|\left\langle\alpha_{+}|P| \beta\right\rangle\right|+\left|\left\langle\alpha_{-}|Q| \beta^{\prime}\right\rangle\right| \\
\leq & \sqrt{\left\langle\alpha_{+}|P| \alpha_{+}\right\rangle} \sqrt{\langle\beta|P| \beta\rangle}+\sqrt{\left\langle\alpha_{-}|Q| \alpha_{-}\right\rangle} \sqrt{\left\langle\beta^{\prime}|Q| \beta^{\prime}\right\rangle} \\
\leq & \sqrt{\left\langle\alpha_{+}|P| \alpha_{+}\right\rangle+\left\langle\alpha_{-}|Q| \alpha_{-}\right\rangle} \sqrt{\langle\beta|P| \beta\rangle+\left\langle\beta^{\prime}|Q| \beta^{\prime}\right\rangle} \\
= & \sqrt{1+\operatorname{Re}\left[\left\langle\alpha|U| \alpha^{\prime}\right\rangle\right]} \sqrt{1+D\left(\sigma_{\mathrm{B}}, \sigma_{\mathrm{B}}^{\prime}\right)} .
\end{aligned}
$$

In a similar manner, $\left|\left\langle\alpha_{+}|Q| \beta\right\rangle\right|+\left|\left\langle\alpha_{-}|P| \beta^{\prime}\right\rangle\right|$ provides a lower bound on $\sqrt{1-\operatorname{Re}\left[\left\langle\alpha|U| \alpha^{\prime}\right\rangle\right]} \sqrt{1-D\left(\sigma_{\mathrm{B}}, \sigma_{\mathrm{B}}^{\prime}\right)}$. We thus obtain

$$
\begin{aligned}
\Delta \leq & \sqrt{\frac{1}{2}+\frac{1}{2} \operatorname{Re}\left[\left\langle\alpha|U| \alpha^{\prime}\right\rangle\right]} \sqrt{\frac{1}{2}+\frac{1}{2} D\left(\sigma_{\mathrm{B}}, \sigma_{\mathrm{B}}^{\prime}\right)} \\
& +\sqrt{\frac{1}{2}-\frac{1}{2} \operatorname{Re}\left[\left\langle\alpha|U| \alpha^{\prime}\right\rangle\right]} \sqrt{\frac{1}{2}-\frac{1}{2} D\left(\sigma_{\mathrm{B}}, \sigma_{\mathrm{B}}^{\prime}\right)},
\end{aligned}
$$

where $\operatorname{Re}\left[\left\langle\alpha|U| \alpha^{\prime}\right\rangle\right]$ in turn provides a lower bound on $F\left(\rho_{\mathrm{E}}, \rho_{\mathrm{E}}^{\prime}\right)$, as in the proof of (12). Substituting $\Delta=\sqrt{1+|\sin (\theta)|} / \sqrt{2}$ and rearranging, we arrive at (3). 
If $\operatorname{dim} \mathcal{H}_{\mathrm{A}}=2$ and the source states form two orthogonal bases, then $\theta$ as defined by (2) coincides with the angle separating the bases on the Bloch sphere. In this particular case, the source is basis independent (i.e. $\frac{1}{2} \rho+\frac{1}{2} \rho^{\prime}=$ $\left.\frac{1}{2} \sigma+\frac{1}{2} \sigma^{\prime}\right)$, and we note that the keyrate (5) is an improvement over the keyrate predicted by the uncertainty relation $[6,7]$, which for comparison is

$$
r \geq 1-\log (1+|\cos (\theta)|)-h\left(\delta_{x}\right)-h\left(\delta_{z}\right) .
$$

A simple example demonstrates that the cloning bound (3) is tight. We set the source states to

$$
\begin{aligned}
|\alpha\rangle & =\cos \left(\frac{\gamma-\theta}{2}\right)|0\rangle_{\mathrm{A}}+\sin \left(\frac{\gamma-\theta}{2}\right)|1\rangle_{\mathrm{A}}, \\
\left|\alpha^{\prime}\right\rangle & =-\sin \left(\frac{\gamma-\theta}{2}\right)|0\rangle_{\mathrm{A}}+\cos \left(\frac{\gamma-\theta}{2}\right)|1\rangle_{\mathrm{A}},
\end{aligned}
$$

and

$$
\begin{aligned}
|\beta\rangle & =\cos \left(\frac{\gamma}{2}\right)|0\rangle_{\mathrm{A}}+\sin \left(\frac{\gamma}{2}\right)|1\rangle_{\mathrm{A}}, \\
\left|\beta^{\prime}\right\rangle & =-\sin \left(\frac{\gamma}{2}\right)|0\rangle_{\mathrm{A}}+\cos \left(\frac{\gamma}{2}\right)|1\rangle_{\mathrm{A}},
\end{aligned}
$$

where $|0\rangle_{\mathrm{A}}$ and $|1\rangle_{\mathrm{A}}$ are orthogonal. Following the cloning operation

$$
\begin{aligned}
|0\rangle_{\mathrm{A}} & \mapsto|0\rangle_{\mathrm{B}}|0\rangle_{\mathrm{E}} \\
|1\rangle_{\mathrm{A}} & \mapsto|1\rangle_{\mathrm{B}}|1\rangle_{\mathrm{E}},
\end{aligned}
$$

we find $D\left(\sigma_{\mathrm{B}}, \sigma_{\mathrm{B}}^{\prime}\right)=|\cos (\gamma)|$ and $F\left(\rho_{\mathrm{E}}, \rho_{\mathrm{E}}^{\prime}\right)=|\sin (\theta-\gamma)|$. If we take, for instance, angles satisfying $0 \leq \gamma \leq \theta \leq \pi / 2$, then $F\left(\rho_{\mathrm{E}}, \rho_{\mathrm{E}}^{\prime}\right)=f_{\theta}\left(D\left(\sigma_{\mathrm{B}}, \sigma_{\mathrm{B}}^{\prime}\right)\right)$. Consequently, the keyrate (5) is the highest that can be derived using the particular approach we have followed so far - i.e. where the conditional entropy is bounded via the fidelity using only the $x$-basis error rate and given only the state characterisation defined in (2). We describe how better bounds on the fidelity and conditional entropy can be derived in certain circumstances of interest in the next sections.

\subsection{Qubit source states}

We now turn to a more detailed study of qubit sources. It will be convenient to characterise such a source in terms of three parameters: an angle $\varphi$ representing the angle between the two bases on the Bloch sphere, and angles $\alpha$ and $\beta$ measuring the non-orthogonality of the states constituting each basis and defined by $|\sin (\alpha)|=\left|\left\langle\alpha \mid \alpha^{\prime}\right\rangle\right|$ and $|\sin (\beta)|=\left|\left\langle\beta \mid \beta^{\prime}\right\rangle\right|$ respectively. We set

$$
\begin{aligned}
& \rho-\rho^{\prime} \propto Z, \\
& \sigma-\sigma \propto V,
\end{aligned}
$$


with $V=\cos (\varphi) Z+\sin (\varphi) X$, and where we can identify $Z$ and $X$ with the Pauli $z$ and $x$ operators, respectively.

Via results from the preceding section, we already know that

$$
\frac{1}{2}\left\|X_{\mathrm{B}}\right\|_{1} \geq f_{\varphi}\left(\frac{1}{2}\left\|V_{\mathrm{B}}\right\|_{1}\right),
$$

where the function $f_{\varphi}$ is as defined in (4), except that we use the angle $\varphi$ between the bases in place of the basis overlap angle $\theta$ defined in (2). If $|\alpha\rangle$ and $\left|\alpha^{\prime}\right\rangle$ are orthogonal, i.e. if $\sin (\alpha)=0$, then according to (12) Eve's fidelity is simply bounded by $F\left(\rho_{\mathrm{E}}, \rho_{\mathrm{E}}^{\prime}\right) \geq \frac{1}{2}\left\|X_{\mathrm{B}}\right\|_{1}$. A natural conjecture one could consider is that this bound still holds if $|\alpha\rangle$ and $\left|\alpha^{\prime}\right\rangle$ are non-orthogonal. This is not the case however: we found counter-examples numerically. Nor is this an artefact of bounding the fidelity as an intermediate step in bounding the keyrate, as we likewise found cases where $H(Z \mid \mathrm{E}) \nsupseteq 1-h\left(\frac{1}{2}+\frac{1}{4}\left\|X_{\mathrm{B}}\right\|_{1}\right)$ when minimising the conditional von Neumann entropy directly.

A generally valid bound, then, will depend explicitly on the non-orthogonality measured by the angle $\alpha$. Specifically, we find

$$
F\left(\rho_{\mathrm{E}}, \rho_{\mathrm{E}}^{\prime}\right) \geq g_{\alpha}\left(\frac{1}{2}\left\|X_{\mathrm{B}}\right\|_{1}\right)
$$

with the function $g_{\alpha}$ defined by

$$
g_{\alpha}(x)=\left\{\begin{array}{ll}
(1+|\sin (\alpha)|) x-|\sin (\alpha)| & : x \geq \frac{2|\sin (\alpha)|}{1+|\sin (\alpha)|} \\
|\sin (\alpha)| & : x \leq \frac{2|\sin (\alpha)|}{1+|\sin (\alpha)|}
\end{array} .\right.
$$

Combining (26) and (27), we have

$$
F\left(\rho_{\mathrm{E}}, \rho_{\mathrm{E}}^{\prime}\right) \geq g_{\alpha} \circ f_{\varphi}\left(\frac{D\left(\sigma_{\mathrm{B}}, \sigma_{\mathrm{B}}^{\prime}\right)}{|\cos (\beta)|}\right),
$$

where we have used that $\sigma-\sigma^{\prime}=\cos (\beta) V$. We see here that explicitly introducing the non-orthogonality of the $x$-basis states can only improve the keyrate bound. This is not surprising since the $x$-basis states are only used for the purpose of testing the channel. Explicitly writing the resulting keyrate,

$$
r \geq 1-h\left[\frac{1}{2}+\frac{1}{2} g_{\alpha} \circ f_{\varphi}\left(\frac{\left|1-2 \delta_{x}\right|}{|\cos (\beta)|}\right)\right]-h\left(\delta_{z}\right) .
$$

The remainder of this section will be devoted to the determination of the function $g_{\alpha}$. First, we set

$$
\begin{aligned}
|\alpha\rangle & =\cos \left(\frac{\alpha}{2}\right)|0\rangle+\mathrm{e}^{\mathrm{i} \phi} \sin \left(\frac{\alpha}{2}\right)|1\rangle, \\
\left|\alpha^{\prime}\right\rangle & =\sin \left(\frac{\alpha}{2}\right)|0\rangle+\mathrm{e}^{\mathrm{i} \phi} \cos \left(\frac{\alpha}{2}\right)|1\rangle,
\end{aligned}
$$


such that $\left\langle\alpha \mid \alpha^{\prime}\right\rangle=\sin (\alpha)$ and $\rho-\rho^{\prime}=\cos (\alpha) Z$. As in preceding sections, we define the Hermitian unitary $U=U_{\mathrm{B}} \otimes \mathbb{1}_{\mathrm{E}}$ such that $\frac{1}{2}\left\|X_{\mathrm{B}}\right\|_{1}=\frac{1}{2} \operatorname{Tr}[U X]=$ $\operatorname{Re}[\langle 0|U| 1\rangle]$. Without loss of generality, we can take the quantity $\Gamma=\langle 0|U| 1\rangle$ to be real (if necessary, this can be achieved by absorbing its phase into the phase $\phi$ already present in (31)). A lower bound is already given by

$$
F\left(\rho_{\mathrm{E}}, \rho_{\mathrm{E}}^{\prime}\right) \geq\left|\left\langle\alpha|U| \alpha^{\prime}\right\rangle\right|,
$$

however the result this would produce is not optimal. In order to obtain a better result, note that any unitary of the form $\tilde{U}_{\mathrm{B}} \otimes \mathbb{1}_{\mathrm{E}}$ can be used in place of $U$ in (32). In particular, since $U$ is Hermitian, the family of operators

$$
U(\gamma)=\mathrm{i} \sin (\gamma) \mathbb{1}+\cos (\gamma) U
$$

are unitary and satisfy this requirement. Evaluating $\left\langle\alpha|U(\gamma)| \alpha^{\prime}\right\rangle$, we find

$$
\begin{aligned}
\left\langle\alpha|U(\gamma)| \alpha^{\prime}\right\rangle= & \frac{1}{2} \sin (\alpha)(\langle 0|U(\gamma)| 0\rangle+\langle 1|U(\gamma)| 1\rangle) \\
& +\mathrm{e}^{\mathrm{i} \phi} \cos \left(\frac{\alpha}{2}\right)^{2}\langle 0|U(\gamma)| 1\rangle+\mathrm{e}^{-\mathrm{i} \phi} \sin \left(\frac{\alpha}{2}\right)^{2}\langle 1|U(\gamma)| 0\rangle \\
= & \mathrm{i} \sin (\gamma) \sin (\alpha)+\cos (\gamma) \sin (\alpha) K \\
& +\cos (\gamma)(\cos (\phi)+\mathrm{i} \cos (\alpha) \sin (\phi)) \Gamma
\end{aligned}
$$

where we have set $K=\frac{1}{2}(\langle 0|U| 0\rangle+\langle 1|U| 1\rangle)$. Squaring the last line,

$$
\begin{aligned}
\left|\left\langle\alpha|U(\gamma)| \alpha^{\prime}\right\rangle\right|^{2}= & \cos (\gamma)^{2}(\sin (\alpha) K+\cos (\phi) \Gamma)^{2} \\
& +(\sin (\gamma) \sin (\alpha)+\cos (\gamma) \cos (\alpha) \sin (\phi) \Gamma)^{2}
\end{aligned}
$$

Our task now is to maximise this quantity over $\gamma$ and minimise over possible values of $K$ and $\phi$. We begin by minimising over $K$ for fixed $\gamma$ and $\phi$. First, note that $|K|+\Gamma \leq 1$ (this can be inferred by evaluating (35) for $\gamma=\phi=0$ and $\alpha= \pm \pi / 2$ and noting that the result should never exceed 1$)$. With this constraint, we wish to minimise $Q=|\sin (\alpha) K+\cos (\phi) \Gamma|$. If $\Gamma$ is sufficiently small, we are able to choose $K$ such that this quantity is zero. Otherwise, we simply set $|K|=1-\Gamma$. Calling $Q_{*}$ the minimum possible value of $|\sin (\alpha) K+\cos (\phi) \Gamma|$, we have

$$
Q_{*}= \begin{cases}(|\sin (\alpha)|+|\cos (\phi)|) \Gamma-|\sin (\alpha)| & : \Gamma \geq \frac{|\sin (\alpha)|}{|\sin (\alpha)|+|\cos (\phi)|} \\ 0 & : \Gamma \leq \frac{|\sin (\alpha)|}{|\sin (\alpha)|+|\cos (\phi)|},\end{cases}
$$

The next step is the maximisation over $\gamma$. Since $Q_{*}$ has no dependence on $\gamma$, this is straightforward. For the optimal value $\gamma_{*}$ of $\gamma$, we find

$$
\begin{aligned}
\left|\left\langle\alpha\left|U\left(\gamma_{*}\right)\right| \alpha^{\prime}\right\rangle\right|^{2}= & \frac{1}{2}\left(Q_{*}{ }^{2}+\cos (\alpha)^{2} \sin (\phi)^{2} \Gamma^{2}+\sin (\alpha)^{2}\right) \\
+\frac{1}{2}\left\{\left(Q_{*}{ }^{2}+\right.\right. & \left.\cos (\alpha)^{2} \sin (\phi)^{2} \Gamma^{2}-\sin (\alpha)^{2}\right)^{2} \\
& \left.+4 \sin (\alpha)^{2} \cos (\alpha)^{2} \sin (\phi)^{2} \Gamma^{2}\right\}^{1 / 2}
\end{aligned}
$$


Finally, we minimise over $\phi$. For values of $\phi$ such that $|\cos (\phi)| \leq|\sin (\alpha)| \frac{1-\Gamma}{\Gamma}$, we set $Q_{*}=0$, and (37) simplifies to

$$
\left|\left\langle\alpha\left|U\left(\gamma_{*}\right)\right| \alpha^{\prime}\right\rangle\right|^{2}=\sin (\alpha)^{2}+\cos (\alpha)^{2} \sin (\phi)^{2} \Gamma^{2} .
$$

If $\Gamma \leq \frac{|\sin (\alpha)|}{1+|\sin (\alpha)|}$, then $Q_{*}=0$ regardless of $\phi$, and the minimum of $(38)$ is

$$
\left|\left\langle\alpha\left|U\left(\gamma_{*}\right)\right| \alpha^{\prime}\right\rangle\right|=|\sin (\alpha)| \text {. }
$$

Otherwise, the minimum of (35) for values of $\phi$ where $Q_{*}=0$ is found by using the minimum allowed value of $|\sin (\phi)|$ in (38), which simplifies to

$$
\left|\left\langle\alpha\left|U\left(\gamma_{*}\right)\right| \alpha^{\prime}\right\rangle\right|=\sin (\alpha)^{2}+\cos (\alpha)^{2} \Gamma \text {. }
$$

If $\Gamma>\frac{|\sin (\alpha)|}{1+|\sin (\alpha)|}$, then we must separately consider the range of possible values of $\phi$ for which $|\cos (\phi)|>|\sin (\alpha)| \frac{1-\Gamma}{\Gamma}$, where $Q_{*}=(|\sin (\alpha)|+|\cos (\phi)|)-$ $|\sin (\alpha)|$. We have

$$
Q_{*}{ }^{2}+\cos (\alpha)^{2} \sin (\phi)^{2} \Gamma^{2}=Y \Gamma(Y \Gamma-2)+2 \cos (\alpha)^{2} \Gamma+\sin (\alpha)^{2},
$$

where we have set $Y=1+|\sin (\alpha)||\cos (\phi)|$. Then,

$$
\begin{aligned}
&\left|\left\langle\alpha\left|U\left(\gamma_{*}\right)\right| \alpha^{\prime}\right\rangle\right|^{2}= \sin (\alpha)^{2}+\cos (\alpha)^{2} \Gamma+\frac{1}{2} Y \Gamma(Y \Gamma-2) \\
&+\frac{1}{2} \Gamma\left\{\left(Y(Y \Gamma-2)+2 \cos (\alpha)^{2}\right)^{2}\right. \\
&\left.+4 \sin (\alpha)^{2} \cos (\alpha)^{2} \sin (\phi)^{2}\right\}^{1 / 2},
\end{aligned}
$$

which we can simplify down to

$$
\begin{aligned}
\left|\left\langle\alpha\left|U\left(\gamma_{*}\right)\right| \alpha^{\prime}\right\rangle\right|^{2}= & \sin (\alpha)^{2}+\cos (\alpha)^{2} \Gamma+\frac{1}{2} Y \Gamma(Y \Gamma-2) \\
& +\frac{1}{2} Y \Gamma \sqrt{(Y \Gamma-2)^{2}+4 \cos (\alpha)^{2}(\Gamma-1)} .
\end{aligned}
$$

This is a decreasing function in $Y$ and is therefore minimised by taking $|\cos (\phi)|=1$, or $Y_{*}=1+|\sin (\alpha)|$. We find

$$
\begin{aligned}
\left|\left\langle\alpha\left|U\left(\gamma_{*}\right)\right| \alpha^{\prime}\right\rangle\right|^{2}= & \sin (\alpha)^{2}+\cos (\alpha)^{2} \Gamma \\
& +\frac{1}{2} Y_{*} \Gamma\left[Y_{*} \Gamma-2+\left|Y_{*} \Gamma-2\right| \sin (\alpha)||\right] .
\end{aligned}
$$

If $\Gamma \geq \frac{2|\sin (\alpha)|}{1+|\sin (\alpha)|}$, then the minimum we find is

$$
\left|\left\langle\alpha\left|U\left(\gamma_{*}\right)\right| \alpha^{\prime}\right\rangle\right|=(1+|\sin (\alpha)|) \Gamma-|\sin (\alpha)| .
$$

Otherwise, the minimum is simply $|\sin (\alpha)|$. Since (45) is always less than (40), our final result is

$$
\left|\left\langle\alpha\left|U\left(\gamma_{*}\right)\right| \alpha^{\prime}\right\rangle\right| \geq\left\{\begin{array}{ll}
(1+|\sin (\alpha)|) \Gamma-|\sin (\alpha)| & : \Gamma \geq \frac{2|\sin (\alpha)|}{1+|\sin (\alpha)|} \\
|\sin (\alpha)| & : \Gamma \leq \frac{2|\sin (\alpha)|}{1+|\sin (\alpha)|}
\end{array} .\right.
$$

Recalling that $F\left(\rho_{\mathrm{E}}, \rho_{\mathrm{E}}^{\prime}\right) \geq\left|\left\langle\alpha\left|U\left(\gamma_{*}\right)\right| \alpha^{\prime}\right\rangle\right|$ and that we defined $\Gamma=\frac{1}{2}\left\|X_{\mathrm{B}}\right\|_{1}$, this concludes the proof of $(27)$. 


\subsection{Qubit source and detector}

In most security analyses of the BB84 protocol accounting for device alignment errors, including the results derived in sections 3.2 and 3.3, only the $x$-basis error rate is used to bound the information an eavesdropper could have about the key. This leaves open the possibility that we could derive better results if the $z$-basis error rate were also used for this purpose. Here, we will illustrate how such a result can be derived in the case where Alice's source emits qubit states and Bob's detector is assumed to perform twodimensional measurements.

As in section 3.3, we take $\rho-\rho^{\prime} \propto Z$ and $\sigma-\sigma^{\prime} \propto V$, where $V=\cos (\varphi) Z+$ $\sin (\varphi) X$, and $Z$ and $X$ can be identified with the Pauli $z$ and $x$ operators. The error rates provide lower bounds on $D\left(\rho_{\mathrm{B}}, \rho_{\mathrm{B}}^{\prime}\right)=|\cos (\alpha)| \frac{1}{2}\left\|Z_{\mathrm{B}}\right\|_{1}$ and $D\left(\sigma_{\mathrm{B}}, \sigma_{\mathrm{B}}^{\prime}\right)=|\cos (\beta)| \frac{1}{2}\left\|V_{\mathrm{B}}\right\|_{1}$. The main intuition for deriving an improved keyrate is that, if $\operatorname{dim} \mathcal{H}_{\mathrm{B}}=2$, the quantities $\frac{1}{2}\left\|Z_{\mathrm{B}}\right\|_{1}, \frac{1}{2}\left\|X_{\mathrm{B}}\right\|_{1}$, and $\frac{1}{2}\left\|V_{\mathrm{B}}\right\|_{1}$ are constrained in the values they can take. Specifically, we will be able to show that

$$
\sqrt{1-\frac{1}{4}\left\|V_{\mathrm{B}}\right\|_{1}^{2}} \geq|\sin (\varphi)| \sqrt{1-\frac{1}{4}\left\|X_{\mathrm{B}}\right\|_{1}^{2}}-|\cos (\varphi)| \sqrt{1-\frac{1}{4}\left\|Z_{\mathrm{B}}\right\|_{1}{ }^{2}} .
$$

Since we have $F\left(\rho_{\mathrm{E}}, \rho_{\mathrm{E}}^{\prime}\right) \geq g_{\alpha}\left(\frac{1}{2}\left\|X_{\mathrm{B}}\right\|_{1}\right)$ from (27), (47) provides a second bound on the fidelity to complement (29). Our end result is similar in form to $(29)$ :

$$
F\left(\rho_{\mathrm{E}}, \rho_{\mathrm{E}}^{\prime}\right) \geq g_{\alpha} \circ f_{\varphi}^{(2)}\left(\frac{D\left(\rho_{\mathrm{B}}, \rho_{\mathrm{B}}^{\prime}\right)}{|\cos (\alpha)|}, \frac{D\left(\sigma_{\mathrm{B}}, \sigma_{\mathrm{B}}^{\prime}\right)}{|\cos (\beta)|}\right),
$$

with the function $f_{\varphi}^{(2)}$ defined piecewise by

$$
f_{\varphi}^{(2)}(z, v)=\left\{\begin{array}{ll}
h_{\varphi}(z, v) & : q(z, v) \geq|\cos (\varphi)| \\
f_{\varphi}(v) & : q(z, v) \leq|\cos (\varphi)|
\end{array},\right.
$$

in turn with $h_{\varphi}$ defined such that

$$
|\sin (\varphi)| \sqrt{1-h_{\varphi}(z, v)^{2}}=\sqrt{1-v^{2}}+|\cos (\varphi)| \sqrt{1-z^{2}},
$$

$f_{\varphi}$ as in (4), and $q$ by

$$
q(z, v)=z v-\sqrt{\left(1-z^{2}\right)\left(1-v^{2}\right)} .
$$

The resulting keyrate is

$$
r \geq 1-h\left[\frac{1}{2}+\frac{1}{2} g_{\alpha} \circ f_{\varphi}^{(2)}\left(\frac{\left|1-2 \delta_{z}\right|}{|\cos (\alpha)|}, \frac{\left|1-2 \delta_{x}\right|}{|\cos (\beta)|}\right)\right]-h\left(\delta_{z}\right) .
$$


We note that, provided $\sin (\varphi) \neq 0$, if $\frac{1}{2}\left\|Z_{\mathrm{B}}\right\|_{1}=1$ and $\frac{1}{2}\left\|V_{\mathrm{B}}\right\|_{1}=1$ then (47) implies $\frac{1}{2}\left\|X_{\mathrm{B}}\right\|_{1}=1$. Thus, except in the pathological case where the $z$ and $x$ bases coincide, if $\delta_{z}=(1-|\cos (\alpha)|) / 2$ and $\delta_{x}=(1-|\cos (\beta)|) / 2$ (the minimum possible error rates) we certify that Bob is in full control of Alice's source space and we find $H(Z \mid \mathrm{E})=1$ and $r \geq 1-h\left(\delta_{z}\right)$ (i.e. the only reduction in the keyrate is due to error correction).

We broke (47) numerically in test cases where $\operatorname{dim} \mathcal{H}_{\mathrm{B}}>2$. In this respect, we see that there is an advantage to be gained if some characterisation of Bob's detector is introduced in addition to Alice's source.

We now prove (47). Our strategy will be to derive an upper bound on $\frac{1}{2}\left\|V_{\mathrm{B}}\right\|_{1}$ given $\frac{1}{2}\left\|Z_{\mathrm{B}}\right\|_{1}$ and $\frac{1}{2}\left\|X_{\mathrm{B}}\right\|_{1}$, and then invert the resulting bound for $\frac{1}{2}\left\|X_{\mathrm{B}}\right\|_{1}$. Where $\mathcal{H}_{\mathrm{B}}$ is two dimensional, this is especially easy as all three operators are expressible as combinations of Pauli operators. We set $Z_{\mathrm{B}}=\boldsymbol{z} \cdot \boldsymbol{\sigma}, X_{\mathrm{B}}=\boldsymbol{x} \cdot \boldsymbol{\sigma}$, and $V_{\mathrm{B}}=\boldsymbol{v} \cdot \boldsymbol{\sigma}$, with $\|\boldsymbol{z}\|,\|\boldsymbol{x}\|,\|\boldsymbol{v}\| \leq 1$. For simplicity of notation we will generally denote by e.g. $a$ the norm $\|\boldsymbol{a}\|$ of a vector $\boldsymbol{a}$. Because $a=\|\boldsymbol{a}\|=\frac{1}{2}\|\boldsymbol{a} \cdot \boldsymbol{\sigma}\|_{1}$, deriving the desired bound is reduced to bounding $v$ in terms of $z$ and $x$. Using that $V_{\mathrm{B}}=\cos (\varphi) Z_{\mathrm{B}}+\sin (\varphi) X_{\mathrm{B}}$, we have

$$
\begin{aligned}
v & =\frac{1}{2}\left\|V_{\mathrm{B}}\right\|_{1} \\
& =\frac{1}{2}\left\|\cos (\varphi) Z_{\mathrm{B}}+\sin (\varphi) X_{\mathrm{B}}\right\|_{1} \\
& =\|\cos (\varphi) \boldsymbol{z}+\sin (\varphi) \boldsymbol{x}\| .
\end{aligned}
$$

Squaring this and developing,

$$
v^{2}=\frac{1}{2}\left(z^{2}+x^{2}\right)+\frac{1}{2} \cos (2 \varphi)\left(z^{2}-x^{2}\right)+\sin (2 \varphi) \boldsymbol{x} \cdot \boldsymbol{z} .
$$

Now, while we are working with a given value of $\varphi$, we can arrive at a nontrivial bound on $v$ by noting that $\frac{1}{2}\left\|\cos (\varphi) Z_{\mathrm{B}}+\sin (\varphi) X_{\mathrm{B}}\right\|_{1}$ must be less than 1 for all values of $\varphi$. The maximum value of (54), if $\varphi$ is allowed to take any value, is given by

$$
\left(v_{\max }\right)^{2}=\frac{1}{2}\left(z^{2}+x^{2}\right)+\sqrt{\frac{1}{4}\left(z^{2}-x^{2}\right)^{2}+(\boldsymbol{x} \cdot \boldsymbol{z})^{2}} .
$$

Requiring that the right-hand side is less than 1, we find

$$
\left(2-z^{2}-x^{2}\right)^{2} \geq\left(z^{2}-x^{2}\right)^{2}+4(\boldsymbol{x} \cdot \boldsymbol{z})^{2},
$$

which simplifies to

$$
|\boldsymbol{x} \cdot \boldsymbol{z}| \leq \sqrt{\left(1-z^{2}\right)\left(1-x^{2}\right)} .
$$

From this, we deduce that

$$
v^{2} \leq \frac{1}{2}\left(z^{2}+x^{2}\right)+\frac{1}{2} \cos (2 \varphi)\left(z^{2}-x^{2}\right)+|\sin (2 \varphi)| \sqrt{\left(1-z^{2}\right)\left(1-x^{2}\right)},
$$


or equivalently,

$$
\sqrt{1-v^{2}} \geq|| \cos (\varphi)\left|\sqrt{1-z^{2}}-\right| \sin (\varphi)\left|\sqrt{1-x^{2}}\right| .
$$

Invoking the triangle inequality on (53), we also trivially have

$$
v \leq|\cos (\varphi)| z+|\sin (\varphi)| x
$$

and

$$
v \geq|| \cos (\varphi)|z-| \sin (\varphi)|x| .
$$

Inverting these, we find two lower bounds on $x$ :

$$
|\sin (\varphi)| \sqrt{1-x^{2}} \leq \sqrt{1-v^{2}}+|\cos (\varphi)| \sqrt{1-z^{2}}
$$

and

$$
|\sin (\varphi)| x \geq|v-| \cos (\varphi)|z|,
$$

and the bound on $x$ we are seeking is simply whichever is the stronger of the two, which depends on $z$ and $v$. Specifically, we should use (62) if $z v-\sqrt{\left(1-z^{2}\right)\left(1-v^{2}\right)} \geq|\cos (\varphi)|$, and (63) otherwise.

Equation (62) is just a rearrangement of (47), and for sufficiently good bounds on $z$ and $v$ provides a better bound on $F\left(\rho_{\mathrm{E}}, \rho_{\mathrm{E}}^{\prime}\right)$ than $(27)$. Equation (63) by contrast proves to be of little interest as in practice we have only lower bounds $z \geq z_{0}$ and $v \geq v_{0}$ on $z$ and $v$. Because (63) is always a decreasing function of either $z$ or $v$, we cannot safely substitute lower bounds $z_{0}$ and $v_{0}$ in their place.

For the sake of completeness, we show here that (62) and (63) together are no more useful than (62) and (27) in this respect. First, note that we certify nothing if $v_{0} \leq|\cos (\varphi)|$, as this permits $v=|\cos (\varphi)|$ and $z=1$, for which both (62) and (63) reduce to $x \geq 0$. If $v_{0}>|\cos (\varphi)|$, then (63) is minimised by maximising $z$ (i.e. setting $z=1$ ) and minimising $v$ (i.e. setting $v=v_{0}$ ). The lower bound on $x$ implied by (62) is minimised by minimising both $z$ and $v$. In both cases we can safely substitute $v=v_{0}$. If $z_{0}$ is such that $z_{0} v_{0}-\sqrt{\left(1-z_{0}^{2}\right)\left(1-v_{0}^{2}\right)} \geq|\cos (\varphi)|$, then we simply use $z=z_{0}$ in (62). Otherwise, we use $z=|\cos (\varphi)| v_{0}+|\sin (\varphi)| \sqrt{1-v_{0}^{2}}$ in either (62) or (63), which both reduce to $x \geq|\sin (\varphi)| v_{0}-|\cos (\varphi)| \sqrt{1-v_{0}^{2}}$.

In summary, given lower bounds $z_{0}$ and $v_{0}$ on $z=\frac{1}{2}\left\|Z_{\mathrm{B}}\right\|_{1}$ and $v=\left\|V_{\mathrm{B}}\right\|_{1}$ respectively, we have shown that

$$
x=\frac{1}{2}\left\|X_{\mathrm{B}}\right\|_{1} \geq f_{\varphi}^{(2)}\left(z_{0}, v_{0}\right),
$$

with the function $f_{\varphi}^{(2)}$ defined in (49) above. This concludes the derivation of (48). 


\section{Conclusion}

We have shown that it is possible to relate the security of the prepare-andmeasure BB84 quantum key distribution protocol in a particularly simple way to the inherent limits of cloning imposed by quantum physics. In this respect, our approach is similar to that explored in $[6,7]$ in that we relate security as closely as possible to a generic characterisation of the structure of quantum physics, with our cloning bounds such as (3) playing a role analogous to the uncertainty relations of $[6,7]$. Notably, in a departure from recent security analyses, we see that a much more direct treatment of the prepareand-measure scenario is possible; recasting BB84 in its entanglement-based form is seen not to be a necessary step prior to the analysis.

Our results hold with Bob's device left largely uncharacterised. Specifically, our results hold wherever the Helstrom bound does, which is at least wherever Bob's measurements are separable. We note this is in line with a trend in recent security analyses featuring automatic "one-sided device independence" [27]. (See also [28] for a recent security proof of BB84 that is completely device independent on Bob's side.)

Our security proof, which is based on the Devetak-Winter bound, holds for collective attacks, in which the eavesdropper is assumed to attack each qubit unitarily individually and identically, but may delay her measurements until after the classical post-processing has been applied. In the case of a BB84 implementation that is basis independent and where Bob's measurement devices are memoryless, security against collective attacks implies security against coherent attacks, as follows for instance from the post-selection technique of [29]. The status of coherent attacks in more general situations is still largely unknown, and we leave as an open question how our results extend in this case.

We have also restricted our analysis to the asymptotic regime (though see section 2.2). To derive a security proof in the case of finite statistics, it would be interesting to understand how our approach can be adapted to bound the smooth min-entropy in a manner that recovers (9) and cloning bounds such as (3) asymptotically. Additionally, as the main purpose of this paper is to introduce an alternative approach to understanding QKD security, we have not attempted to account for all possible issues that should be accounted for in a truly unconditional security proof of practical QKD. In particular, for simplicity we have assumed a lossless channel and perfect detector efficiency. While this restriction can in principle be removed if Bob adopts the convention of assigning outputs to non-detection events, in practice the resulting effective error rates would be too high to certify the 
security of a QKD scheme implemented with contemporary technology, and a better treatment of losses and detector inefficiencies seems desirable. We refer to [11] for a possible approach treating non-detection as a third possible outcome.

Finally, following the recent trend of partially or fully device-independent approaches to QKD [30-32], let us remark that our security proof of BB84 depends, as in [11], on a single parameter $\theta$ characterising the quantum devices. Provided that our techniques generalise to coherent attacks, this parameter would represent the only feature that has to be trusted in an otherwise device-independent implementation of BB84. It would be interesting then to understand how this parameter could be "self tested" under some realistic assumptions, hopefully then elevating the prepare-and-measure BB84 protocol to a status competitive with existing partially device-independent approaches to QKD.

\section{Acknowledgements}

The author thanks Stefano Pironio for useful discussion and very helpful suggestions and guidance during the preparation of this manuscript, and acknowledges support from the Belgian Fonds pour la Formation à la Recherche dans l'Industrie et dans l'Agriculture (F.R.I.A.), the EU projects QEssence and QCS, the CHIST-ERA DIQIP project, the Interuniversity Attraction Poles Photonics@be Programme (Belgian Science Policy), and the FRS-FNRS under project DIQIP.

\section{References}

[1] C. H. Bennett and G. Brassard, in Proceedings of IEEE International Conference on Computers, Systems and Signal Processing, Vol. 11 (IEEE, New York, 1984) pp. 175-179.

[2] A. K. Ekert, Phys. Rev. Lett. 67, 661 (1991).

[3] C. H. Bennett, G. Brassard, and N. D. Mermin, Phys. Rev. Lett. 68, 557 (1992).

[4] M. Koashi and J. Preskill, Phys. Rev. Lett. 90, 057902 (2003).

[5] E. Woodhead and S. Pironio, Phys. Rev. A 87, 032315 (2013).

[6] M. Berta, M. Christandl, R. Colbeck, J. M. Renes, and R. Renner, Nat. Phys. 6, 659 (2010).

[7] M. Tomamichel and R. Renner, Phys. Rev. Lett. 106, 110506 (2011). 
[8] M. Tomamichel, C. C. W. Lim, N. Gisin, and R. Renner, Nat. Commun. 3, 634 (2012).

[9] D. Gottesman, H.-K. Lo, N. Lütkenhaus, and J. Preskill, Quantum Inf. Comput. 4, 325 (2004).

[10] M. Koashi, New J. Phys. 11, 045018 (2009).

[11] O. Marøy, L. Lydersen, and J. Skaar, Phys. Rev. A 82, 032337 (2010).

[12] W. K. Wootters and W. H. Zurek, Nature 299, 802 (1982).

[13] D. Dieks, Phys. Lett. A 92, 271 (1982).

[14] V. Scarani, S. Iblisdir, N. Gisin, and A. Acín, Rev. Mod. Phys. 77, 1225 (2005).

[15] D. Mayers, J. ACM 48, 351 (2001).

[16] E. Biham, M. Boyer, G. Brassard, J. van de Graaf, and T. Mor, Algorithmica 34, 372 (2002).

[17] C. A. Fuchs, N. Gisin, R. B. Griffiths, C.-S. Niu, and A. Peres, Phys. Rev. A 56, 1163 (1997).

[18] P. W. Shor and J. Preskill, Phys. Rev. Lett. 85, 441 (2000).

[19] W.-Y. Hwang, Phys. Rev. Lett. 91, 057901 (2003).

[20] H.-K. Lo, X. Ma, and K. Chen, Phys. Rev. Lett. 94, 230504 (2005).

[21] I. Devetak and A. Winter, Proc. R. Soc. A 461, 207 (2005).

[22] C. W. Helstrom, Quantum detection and estimation theory (Academic Press, New York, 1976).

[23] W. Roga, M. Fannes, and K. Życzkowski, Phys. Rev. Lett. 105, 040505 (2010).

[24] M. Tomamichel, A Framework for Non-Asymptotic Quantum Information Theory, Ph.D. thesis, ETH Zurich (2012), arXiv:1203.2142 [quant$\mathrm{ph}$.

[25] R. König, R. Renner, and C. Schaffner, IEEE Trans. Inf. Th. 55, 4337 (2009).

[26] E. Woodhead, C. C. W. Lim, and S. Pironio, in Theory of Quantum Computation, Communication, and Cryptography, Lecture Notes in Computer Science, Vol. 7582 (Springer Berlin Heidelberg, 2013) pp. $107-115$.

[27] C. Branciard, E. G. Cavalcanti, S. P. Walborn, V. Scarani, and H. M. Wiseman, Phys. Rev. A 85, 010301 (2012).

[28] M. Tomamichel, S. Fehr, J. Kaniewski, and S. Wehner, in Advances in Cryptology - EUROCRYPT 2013, Lecture Notes in Computer Science, Vol. 7881 (Springer Berlin Heidelberg, 2013) pp. 609-625.

[29] M. Christandl, R. König, and R. Renner, Phys. Rev. Lett. 102, 020504 (2009).

[30] A. Acín, N. Brunner, N. Gisin, S. Massar, S. Pironio, and V. Scarani, Phys. Rev. Lett. 98, 230501 (2007).

[31] M. Pawłowski and N. Brunner, Phys. Rev. A 84, 010302 (2011).

[32] H.-K. Lo, M. Curty, and B. Qi, Phys. Rev. Lett. 108, 130503 (2012). 